\title{
Heat Shock Protein Member 8 Is an Attachment Factor for Infectious Bronchitis Virus
}

\author{
Pengpeng Zhut, Chenfei Lv', Chengxiu Fang, Xing Peng, Hao Sheng, Peng Xiao, \\ Nishant Kumar Ojha, Yan Yan, Min Liao* and Jiyong Zhou
}

Key Laboratory of Animal Virology of Ministry of Agriculture, Zhejiang University, Hangzhou, China

OPEN ACCESS

Edited by:

Akio Adachi,

Kansai Medical University, Japan

Reviewed by:

Rodrigo A. Gallardo,

University of California, Davis,

United States

Tomomi Takano,

Kitasato University, Japan

Vicky Lin Van Santen,

Auburn University, United States

*Correspondence:

Min Liao

liaomin4545@zju.edu.cn

${ }^{\dagger}$ These authors have contributed equally to this work

Specialty section:

This article was submitted to

Virology,

a section of the journal

Frontiers in Microbiology

Received: 06 March 2020

Accepted: 22 June 2020

Published: 10 July 2020

Citation:

Zhu P, Lv C, Fang C, Peng $X$, Sheng H, Xiao P, Kumar Ojha N, Yan Y, Liao M and Zhou J (2020) Heat Shock Protein Member 8 Is an Attachment Factor for Infectious

Bronchitis Virus.

Front. Microbiol. 11:1630. doi: 10.3389/fmicb.2020.01630
Although infectious bronchitis virus (IBV) is the first coronavirus identified, little is known about which membrane protein of host cells could interact with IBV spike protein and facilitate the infection by the virus. In this study, by using a monoclonal antibody to the S1 protein of IBV M41 strain, we found that heat shock protein member 8 (HSPA8) could interact with spike protein of IBV. HSPA8 was found to be present on the cell membrane and chicken tissues, with highest expression level in the kidney. Results of co-IP and GST-pull-down assays indicated that the receptor binding domain (RBD) of IBV M41 could interact with HSPA8. The results of binding blocking assay and infection inhibition assay showed that recombinant protein HSPA8 and antibody to HSPA8 could inhibit IBV M41 infection of chicken embryonic kidney (CEK) cells. Further, we found that HSPA8 interacted with the N-terminal 19-272 amino acids of S1 of IBV Beaudette, H120 and QX strains and HSPA8 from human and pig also interacted with IBV M41-RBD. Finally the results of binding blocking assay and infection inhibition assay showed that recombinant HSPA8 protein and antibody to HSPA8 could inhibit IBV Beaudette strain infection of Vero cells that were treated with heparanase to remove heparan sulfate from the cell surface. Taken together, our results indicate that HSPA8 is a novel host factor involved in IBV infection.

Keywords: infectious bronchitis virus, HSPA8, virus entry, receptor binding domain, attachment factor

\section{INTRODUCTION}

Infectious bronchitis virus (IBV) is a member of genus gamma-coronavirus in the family Coronaviridae, causing serious economic losses to the poultry industry (Cavanagh, 2007). IBV has many genotypes and serotypes circulating in poultry farms leading to continuous outbreaks of infectious bronchitis (IB) disease (Bijlenga et al., 2004). IBV is an enveloped virus with a single stranded unsegmented positive sense RNA genome of about $27 \mathrm{~kb}$ size. The IBV virion is made up of spike protein $(\mathrm{S})$, membrane protein $(\mathrm{M})$, nucleoprotein $(\mathrm{N})$, envelope protein $(\mathrm{E})$, and genomic RNA (Lai and Cavanagh, 1997). Spike protein determines the IBV tropism and can be cleaved into the two subunits: S1 and S2 by host furin protein (Cavanagh et al., 1992; Casais et al., 2003). The S1 subunit is responsible for binding to the cell surface receptor, while S2 is responsible for fusion of viral envelope and cellular membrane (Luo and Weiss, 1998; Belouzard et al., 2012).

Interaction between virus protein and cellular receptor is the first step for virus infection (Smith and Helenius, 2004). Different coronaviruses use different cell surface molecules to gain entry into 
the target cells. For instance, Aminopeptidase N (APN) is the receptor for H229E, TGEV, and PDCoV (Delmas et al., 1992; Breslin et al., 2003; Wang and Huang, 2019); MHV utilizes CEACAM1 as receptor (Williams et al., 1991); ACE2 is the receptor for SARS-CoV (Li et al., 2003) and recently emerged SARS-CoV-2 (Wan et al., 2020); DPP4 is the receptor for MERSCoV (Raj et al., 2013). Meanwhile, besides cellular receptors, other co-factors in the cell membrane may also influence virus binding. For example, glucose-regulated protein 78 (GRP-78) is an important attachment factor for MERS-CoV infection of host cells (Chu et al., 2018). Calcium dependent (C-type) lectins play a role during SARS-CoV and FCoV infection (Belouzard et al., 2012).

Though IBV was the first coronavirus discovered, study of its host cell receptor goes slowly. Previous studies showed that $\alpha$ 2,3 sialic acid and heparan sulfate (HS) were binding molecules for IBV onto the host cells (Winter et al., 2006; Madu et al., 2007). Recently, Wang et al. reported that IBV entered the cells through clathrin-mediated endocytosis (Wang et al., 2019). The $\mathrm{N}$ terminal 253 amino acids of the mature S1 subunit of IBV-M41 (aa 19-272 of the S1 precursor) has been identified as a receptor binding domain (RBD) (Promkuntod et al., 2014). However, the functional receptor for IBV infection is still unknown. In this study, we attempt to identify molecules on host cell membrane responsible for IBV infection. Using immunoprecipitation assay and mass spectrometry analysis, we identified heat shock protein member 8 (HSPA8) as a host cell molecule involved in IBV infection. HSPA8 is a member of HSP70 family and is also referred to as HSP71. It is a conserved protein among different species during evolution (Stricher et al., 2013). A large number of cellular functions have been attributed to HSPA8 (Liu et al., 2012). Previous research showed that HSPA 8 can exist in cell membrane and has a role in binding to cell surface by several viruses such as Rotavirus and Human T-Cell Lymphotropic Virus Type 1 (Sagara et al., 1998; Guerrero and Moreno, 2012). Our results suggest that HSPA8 plays a role during initial stages of IBV infection.

\section{MATERIALS AND METHODS}

\section{Cells, Viruses, Antibodies, Reagents, SPF Embryonated Eggs, and Chickens}

Chicken embryo kidney (CEK) cells were prepared from 17 to 19 day-old SPF embryonated chicken eggs and maintained in DMEM supplemented with $10 \% \mathrm{FBS}$ at $37^{\circ} \mathrm{C}$ in $5 \% \mathrm{CO}_{2}$ atmosphere. Vero and 293T cells were maintained in DMEM supplemented with $10 \% \mathrm{FBS}$ at $37^{\circ} \mathrm{C}$ in $5 \% \mathrm{CO}_{2}$ atmosphere. IBV M41 (GenBank: MK937830.1), H120 (GenBank: MK937831.1) and QX vaccine strains (GenBank: KY933090.1) were purchased from China Institute of Veterinary Drug Control and stored in our laboratory. IBV Beaudette strain was a gift from Prof. DingXiang Liu from South China Agricultural University, Guangdong, P.R. of China. Culture of IBV virus was carried out in bio-safety cabinet (SG603A, BAKER) in bio-safety level 2 plus $($ BSL2 +) laboratory.

In-house mAbs against IBV S (1H1), M (2B3) and N (4C1) proteins were produced by our research group by the method described previously ( $\mathrm{Hu}$ et al., 2007). mAb against HSPA8 (ab19136, mouse) and Actin (ab179467, rabbit) were purchased from Abcam. Rabbit IgG (A7016) and mouse IgG (A7028) were purchased from the Beyotime Institute of Biotechnology. mAbs against HSP90AB1 (EM21103, mouse), GAPDH (EM1101, rabbit), GST (EM80701, mouse), GFP (ET1602-7, rabbit), His (R1207-2, mouse), Histone3 (M1306-4, mouse) and polyclonal antibody against HSPA8 (R1511-6, rabbit) were purchased from Huaan Biological Technology. mAb against Flag was purchased from Sigma-Aldrich (F1804, mouse). The secondary antibodies (horseradish peroxidase [HRP]-labeled anti-mouse or anti-rabbit IgG) used for Western blotting were purchased from KPL (Millford, MA). NP-40 lysis buffer (P0013F) was purchased from the Beyotime Institute of Biotechnology. Glutathione-Sepharose beads were purchased from Thermo Fisher Scientific. Ni-NTA agarose (30210) was purchased from QIAGEN. Heparanase I (S31310) was purchased from the Shanghai Yuanye Biological Technology. Specific pathogen free (SPF) chicken embryonated eggs and one-day-old chickens were purchased from Ningbo Chunpai Agricultural Technology Company. The use of chicken tissues in this study was approved by the Committee on the Ethics of Animal of Zhejiang University (ZJU20170388).

\section{Plasmid Construction and Transfection}

HSPA8 gene fragments of different species were amplified from genomic DNA of CEK (chicken), 293T (human) and PK15 (pig) cells by PCR with primers Chicken-HSPA8-F/R, Human-HSPA8$\mathrm{F} / \mathrm{R}$ and Pig-HSPA8-F/R listed in Table 1. The fragments were then cloned into the pCMV-flag- $\mathrm{N}$ vector between restriction enzyme EcoRI and KpnI sites by homologous recombination. M41-S1-RBD (253), Beaudette-S1-253, H120-S1-253, QX-S1253 gene fragments (the N-terminal 19-272 amino acids of the S1 subunit) were amplified from total RNA extracted from individual specific virus strain infected cells or infected chicken embryo allantoic fluid by RT-PCR with primers M41-S1RBD(253)-F/R, Beaudette-S1-253-F/R, H120-S1-253-F/R, and QX-S1-253-F/R listed in Table 1. The gene fragments were cloned into pEGFP-C3 vector between restriction enzyme EcoRI and BamHI sites by homologous recombination.

\section{Immunoprecipitation and Co-immunoprecipitation Assays}

The membrane proteins from IBV M41 infected CEK cells were extracted using Membrane protein extraction kit (Beyotime) following the manufacturer's protocol. Membrane protein extracts were immunoprecipitated (IP) with mAbs $1 \mathrm{H} 1$ against IBV M41 S protein, as well as $\mathrm{mAb}$ against mouse IgG. The precipitated proteins were detected by silver staining and Western blotting using anti-S and anti-HSPA8 antibodies. 293T cells were cotransfected with flag-n/EGFP-C3-M41RBD, flag-n-HSPA8/EGFP-C3-M41-RBD, flag-n-HSPA8/EGFPC3-BD-253, flag-n/EGFP-C3-BD-253, flag-n-HSPA8/EGFP-C3H120-253, flag-n/EGFP-C3-H120-253, flag-n-HSPA8/EGFP-C3QX-253, flag-n/EGFP-C3-QX-253, flag-human-HSPA8/EGFPC3-M41-253 and flag-pig-HSPA8/EGFP-C3-M41-253 and then flag-tagged HSPA8 was immunoprecipitated with flag antibody 
TABLE 1 | Primers used for gene amplification in this study.

\begin{tabular}{|c|c|}
\hline Primers & Sequence $\left(5^{\prime}-3^{\prime}\right)$ \\
\hline Chicken-HSPA8-F & tggccatggaggcccgaattcGGATGTCAAAGGGACCAGCTGTTG \\
\hline Chicken-HSPA8-R & gatccccgcggccgcggtacc TТAАТССАССТССТСАATGGTTG \\
\hline Human-HSPA8-F & tggccatggaggcccgaattcATGTCCAAGGGACCTGCAGT \\
\hline Human-HSPA8-R & gatccccgcggccgcggtacc TTAATCAACСТCTTCAATGGTGG \\
\hline Pig-HSPA8-F & tggccatggaggcccgaattcGGATGTCTAAGGGACCTGCAGT \\
\hline Pig-HSPA8-R & gatccccgcggccgcggtacc TAGTCAACСTCCTCAATGG \\
\hline M41-S1-RBD(253)-F & tcgagctcaagcttcgaattcTGATGGCTITGTATGACAGTAGTTCT \\
\hline M41-S1-RBD(253)-R & ttatctagatccggtggatccTCAATTGTGTAACGTAAAAGTAGTATT \\
\hline Beaudette-S1-253-F & tcgagctcaagcttcgaattcTGATGGCTITGTATGACAGTAGTCT \\
\hline Beaudette-S1-253-R & ttatctagatccggtggatccTCAATTGTGTAACGTAAAAGTAGTATT \\
\hline H120-S1-253-F & tcgagctcaagcttcgaattcTGATGGCTITGTATGACAGTAGTTCT \\
\hline H120-S1-253-R & ttatctagatccggtggatccTCAAAACCACAAGCCATTATTA \\
\hline QX-S1-253-F & tcgagctcaagcttcgaattcTGATGAATTGTITGATTCTGATAATA \\
\hline QX-S1-253-R & ttatctagatccggtggatccTCACGCCAGAGTAGTATTAACACTA \\
\hline
\end{tabular}

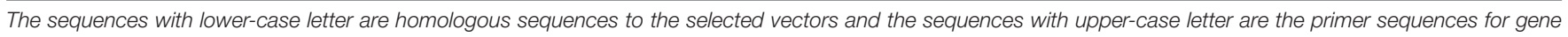
amplification.

and captured by Sepharose A/G beads. The precipitated proteins were then detected by Western blotting using antiflag and anti-GFP antibodies. On the other hand, 293T cells were cotransfected with flag-n-HSPA8/EGFP-C3-M41RBD and flag-n-HSPA8/EGFP-C3 and then GFP-tagged M41RBD immunoprecipitated with GFP antibody and captured by Sepharose A/G beads. The precipitated proteins were then detected by Western blotting using anti-flag and antiGFP antibodies.

\section{Western Blot Analysis}

Cell lysates and immunoprecipitated proteins in protein loading buffer were subjected to SDS-PAGE and transferred onto a nitrocellulose membrane. After being blocked with 5\% skimmed milk, the membranes were incubated with the indicated primary antibodies at $4^{\circ} \mathrm{C}$ overnight. After three washes with PBS, the membranes were incubated with HRP-labeled secondary antibody at room temperature for $1 \mathrm{~h}$. The protein bands were then visualized using enhanced chemiluminescence reagent and imaged using AI680 Imager (GE Healthcare).

\section{Mass Spectrometry}

Specific band in silver stained gel was cut and sent for commercial mass spectrometry (LC-MS/MS) analysis carried out by Shanghai Applied Protein Technology Company.

\section{Indirect Flow Cytometry}

CEK, Vero and 293T cells were cultured in two $10 \mathrm{~cm}$ dishes and were harvested and washed three times with ice cold PBS. Then, $1 \mu \mathrm{g} / \mathrm{ml}$ of the mAb against HSPA8 (ab19136) was added into the cell suspensions and incubated for $30 \mathrm{~min}$ at $4^{\circ} \mathrm{C}$. After that, the cells were washed 3 times by repeat centrifugation at $400 \mathrm{~g}$ for $5 \mathrm{~min}$ and the final pellets were resuspended in ice cold PBS. The fluorochrome-labeled secondary antibody was diluted (1:400) in $3 \%$ BSA/PBS and mixed with above cell suspension and again incubated for $30 \mathrm{~min}$ at $4^{\circ} \mathrm{C}$ in the dark. The cells were washed 3 times by centrifugation at $400 \mathrm{~g}$ for $5 \mathrm{~min}$ and resuspended in ice cold PBS. Finally, samples were analyzed by the flow cytometer (Beckman coulter FC500).

\section{GST Pull Down Assay}

GST, GST-M41-RBD, GST-HSPA8, and His-HSPA8 recombinant proteins were expressed in Escherichia coli BL-21 and then purified using glutathione-sepharose beads and Ni-NTA agarose beads according to the manufacturer's protocol. GST, GSTM41-RBD, and GST-HSPA8 recombinant proteins were bound to glutathione-sepharose beads at $4^{\circ} \mathrm{C}$ for $4 \mathrm{~h}$. After washing five times with PBS, the beads were incubated for $12 \mathrm{~h}$ at $4^{\circ} \mathrm{C}$ with His-HSPA8 protein and EGFP-C3-M41-RBD cell lysates. After washing the beads five times, the protein complex was dissociated from the beads by boiling with 4xSDS-PAGE loading buffer for $10 \mathrm{~min}$, run on SDS-PAGE, and subjected to Western blot analysis using antibodies against GST tag, 6xHis tag and GFP tag.

\section{RT-qPCR}

Total cellular RNA was isolated by the Trizol reagent (Vazyme) according to the manufacturer's instructions. One microgram of total RNA was transcribed into cDNA using a reverse transcription kit (Vazyme). The relative abundance of viral RNA and mRNAs was analyzed using the ChamQ Universal SYBR RT-qPCR master mix (Vazyme) and the LightCycler 96 sequence detector system (Roche). Primers 5'-GAAGAAAACCAGTCCCAGA-3' and $5^{\prime}$-TTACCAGCAACCCACAC-3' were used to detect IBV viral RNA (Kint et al., 2016), the primers 5'-CATCACAGCCAC ACAGAAG- $3^{\prime}$ and 5'-GGTCAGGTCAACAACAGAGA-3' were used to detect chicken GAPDH mRNA (Kint et al., 2016). The primers 5'-GATCTGGCACCACACCTTCT-3' and 5'-GGGGTGTTGAAGGTCTCAAA-3' were used to detect African green monkey $\beta$-actin mRNA (Wang et al., 2019). The primers $5^{\prime}$-TGACCAGGGTAACAGGACCA-3' 
and $5^{\prime}$-ACGCCCAATCAACCGTTTTG-3' were used to detect chicken HSPA8 mRNA.

\section{Binding Blocking Assay}

IBV M41 $\left(\right.$ TCID $\left._{50}=10^{6.5} / \mathrm{ml}, 200 \mu \mathrm{l}\right)$ was incubated with recombinant protein GST-HSPA8 $(100 \mu \mathrm{g})$ and control recombinant protein GST $(100 \mu \mathrm{g})$ at $37^{\circ} \mathrm{C}$ for $1 \mathrm{~h}$. CEK cells cultured in 6-well plates were incubated with GST-HSPA8treated virus and GST-treated virus at $4^{\circ} \mathrm{C}$ for $1 \mathrm{~h}$. After the incubation, the cells were harvested after 3 washes with PBS. Cell-associated viral RNA was quantified by RT-qPCR which indicated the degree of inhibition of virus binding on the host cells caused by recombinant HSPA8. IBV-Beaudette strain $($ moi $=10)$ was incubated with recombinant GSTHSPA8 $(100 \mu \mathrm{g})$ protein and control protein GST $(100 \mu \mathrm{g})$ at $37^{\circ} \mathrm{C}$ for $1 \mathrm{~h}$. Before addition of virus to the Vero cells, $400 \mu \mathrm{l}$ of heparanase I $(5 \mathrm{mIU} / \mathrm{ml})$ was added to the cells and incubated at $37^{\circ} \mathrm{C}$ for $1 \mathrm{~h}$. After washing with PBS, the cells were then incubated with GST-HSPA8-treated virus and GST-treated virus at $4^{\circ} \mathrm{C}$ for $1 \mathrm{~h}$. After incubation, the cells were harvested after 3 washes with PBS. Cell-associated viral RNA was quantified by RT-qPCR which indicated the inhibition level of recombinant HSPA8 on IBV Beaudette binding to the Vero cells.

\section{Infection Inhibition Assay}

CEK cells were cultured in 6-well plates, and incubated with polyclonal antibody against $\operatorname{HSPA} 8(2 \mu \mathrm{g}, 2 \mu \mathrm{l} /$ well or $4 \mu \mathrm{g}$, $4 \mu \mathrm{l} /$ well $)$ and rabbit-IgG $(4 \mu \mathrm{g}, 4 \mu \mathrm{l} /$ well $)$ at $37^{\circ} \mathrm{C}$ for $1 \mathrm{~h}$. The cells were then incubated with IBV M41 $\left(\mathrm{TCID}_{50}=10^{6} .5 / \mathrm{ml}, 50\right.$ $\mu \mathrm{l} /$ well) at $4^{\circ} \mathrm{C}$ for $1 \mathrm{~h}$, after three washes with PBS, the cells were then transferred to incubate at $37^{\circ} \mathrm{C}$ for $1 \mathrm{~h}$ or $24 \mathrm{~h}$. The cells were harvested 1 hpi or 24 hpi after 3 washes with PBS and subjected to RT-qPCR ( 1 and $24 \mathrm{hpi}$ ) and Western blot analysis (24 hpi). The culture supernatants were also collected $24 \mathrm{hpi}$ for TCID 50 assay. For inhibition assay on IBV Beaudette strain, Vero cells were cultured in 6-well plates and incubated with $400 \mu \mathrm{l}$ of heparinase I $(5 \mathrm{mIU} / \mathrm{ml})$ at $37^{\circ} \mathrm{C}$ for $1 \mathrm{~h}$. After 3 washes with PBS, the cells were then incubated with polyclonal antibody against HSPA8 $(2 \mu \mathrm{g}, 2 \mu \mathrm{l} /$ well or $4 \mu \mathrm{g}, 4 \mu \mathrm{l} /$ well $)$ and rabbit-IgG $(4 \mu \mathrm{g}, 4 \mu \mathrm{l} /$ well $)$ at $37^{\circ} \mathrm{C}$ for $1 \mathrm{~h}$. The cells then incubated with IBV Beaudette strain $(\mathrm{moi}=1)$ at $4^{\circ} \mathrm{C}$ for $1 \mathrm{~h}$, after three washes with PBS, the cells were then transferred to incubate at $37^{\circ} \mathrm{C}$ for 1 or $24 \mathrm{~h}$. Similarly, the cells were harvested 1 or 24 hpi after 3 washes with PBS and subjected to RT-qPCR (1 and $24 \mathrm{hpi}$ ) and Western blot analysis (24 hpi). The culture supernatants were also collected $24 \mathrm{hpi}$ for $\mathrm{TCID}_{50}$ assay.

\section{Statistical Analysis}

All data are presented as means \pm standard deviations (SDs) and analyzed by GraphPad. Significant differences between two groups were analyzed using Student's $t$-test. $P$-values of differences between means are represented in figures as follows: ${ }^{* * *} P<0.001$; ${ }^{* *} P<0.01 ;{ }^{*} P<0.05$; and ns (nonsignificant), $P>0.05$.

\section{RESULTS}

\section{HSPA8 Was Identified as Cell Membrane Protein That Interacts With Spike Protein of IBV}

Up to now, little is known about which membrane proteins of CEK cells could interact with IBV spike protein and facilitate the infection by IBV. To address this question, the IBV-M41 strain was passaged in CEK cells to get a CEK culture adapted IBV M41 virus strain. As showed by Western blot analysis of the viral proteins, the expression level of S1 protein of IBV M41 reached a peak at $36 \mathrm{~h}$ post-infection (Figure 1A), which was selected as the optimal time point for preparation of immunoprecipitated S protein in the following experiments. Western blot analysis showed that the membrane proteins could be efficiently extracted with membrane protein extraction kit as the extracted proteins could react with antibody to membrane protein marker HSP90AB1 but not with antibodies to a cytoplasmic protein (GAPDH) and a nuclear protein (Histone3) (Figure 1E).

To identify membrane proteins that may interact with IBV spike protein, immunoprecipitation assay was performed with membrane proteins from IBV M41-CEK infected cells using $\mathrm{mAb}$ $1 \mathrm{H} 1$ to IBV spike protein and mouse IgG. Immunoprecipated proteins were resolved by SDS-PAGE before silver staining (Figure 1B). A specific protein band with molecular weight of $70 \mathrm{kDa}$ (arrow) was visualized by silver staining in $1 \mathrm{H} 1$ immunoprecipitates from infected cells (Figure 1B, lane 3), but not from uninfected cells (not shown). IBV S protein around $170 \mathrm{kDa}$ and its S1 subunit around $100 \mathrm{kDa}$ were detected by Western blot analysis with mAb 1H1 to IBV M41 S1 (Figure 1C, red arrows). The $70 \mathrm{kDa}$ specific protein band (Figure 1B, lane 3 , arrow) which was pulled down by $\mathrm{mAb}$ to $\mathrm{S} 1$ (1H1) but not by the control antibody was excised and sent for LC-MS/MS analysis. The MS result revealed that the specific $70 \mathrm{kDa}$ band was heat shock protein member 8 (HSPA8), also known as heat shock protein 71 . The full length sequence of putative chicken HSPA8 is 647 amino acid in size (Figure 1D). Chicken HSPA8 could be detected from whole cell lysates and membrane extracts of CEK cells (Figure 1E).

\section{HSPA8 Was Further Confirmed to Be Expressed on the Surface of Host Cells and in Chicken Tissues}

To verify whether HSPA8 is expressed on the cell surface, we performed indirect flow cytometry assay without cell permeabilization. As results shown in Figures 2A,C,E, HSPA8 was found to be expressed on the surface of 293T, Vero and CEK cells. Additionally, the expression was also confirmed by Western blot analysis with $\mathrm{mAb}$ to HSPA8 (Figures 2B,D,F).

Furthermore, we checked the expression of HSPA8 in chicken tissues by RT-qPCR. The results showed that HSPA8 was found to be expressed in the tissues of kidney, liver, heart and lung of 1-day-old chickens with highest expression level in kidneys (Figure 2G). 
A

Oh $12 \mathrm{~h} 24 \mathrm{~h} 36 \mathrm{~h} 48 \mathrm{~h}$

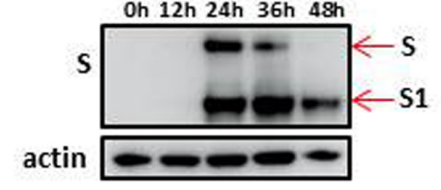

C

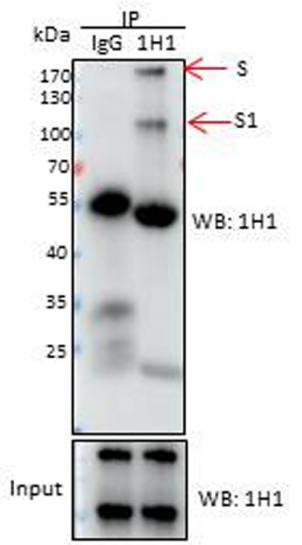

B

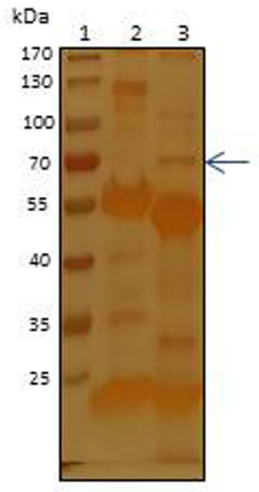

D

1-5OMSKGPAVGIDLGTTYSCVGVFQHGKVEIIANDRGNRTTPSYVAFTOTERL S1-10OLGDAAKNQVAMNPTNTVFDAKRLIGRRFDOSVVQSOMKHWPFTVVNOAGR 101-150PKVQVEYKGE TKSFYPEEISSMVITKMKEIAEAYLGKTUTNAVVTVPAYF 151-200NOSQRQGTKDAGTIAGLNVLRIINEPTAAAIAYGLOKKVGAERNLLIFOL 201.25OGGGTFDVSULTIENGIFEVKSTAGDTHLGGEDFONRLVNHFIAEFKRKHX 215-30OKDISEN KRAVRRLRTACERAKRTLSSSTQASIEIDSLYEGIDFYTSITRA 301-35ORFEKLNADLFRGLOPVEKALRDAKLOKSQIHDIVIVGGSTRIPKIQKLL 351.40OQDFFNGKELNKSINPDEAVAYGAAVQAALLSDKSENVQDLLLLDVTPLS 401-45OLGIETAGGVMTVLIKENTTIPTKQTQTETTYSONQPGVLIQVYEGERAMT 4S1.50OKDNNLLGKFELTGIPPAPRGVPQIEVTFDIOANGILNVSAVOKSTGKEN K. 501.55OITITNDKGRLSKEDIERMVQ EAEKYKAEDEKQRDXVSSKNSLOSYAFNMK 551.600ATVEDEKLPGXILOEOHQNILDXCNEHNWLOKNQTAEKEEFEHQQXELE 601.647 KYCNPUIKLYQSAGGMPGGMPGGFPGGGAPPSG GASSGPTIEEVD

E

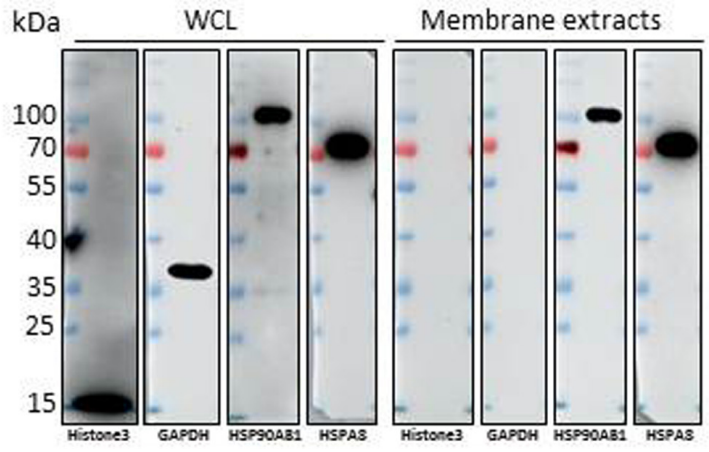

FIGURE 1 | Identification of HSPA8 as a target membrane molecule for IBV Spike protein. (A) Determination of viral protein expression level of IBV M41 strain in CEK cells. CEK cells were infected with IBV M41 (TCID $50=10^{6.5} / \mathrm{ml}, 50 \mu \mathrm{l} /$ well) in 6 -well plates. Infected cells were harvested at 0, 12, 24, 36, and 48 h post-infection. The whole cell lysates were subjected to SDS-PAGE and Western blot analysis with $\mathrm{mAb} 1 \mathrm{H} 1$ to spike protein. The expression of cellular actin protein was used as control. (B) Silver staining of membrane proteins of CEK cells after immunoprecipitation assay. Membrane protein extracts of IBV M41 infected CEK cells were immunoprecipitated with mAb $1 \mathrm{H} 1$ (lane 3) to IBV M41 spike protein, and mouse IgG (lane 2). Lane 1, protein marker. Arrow indicated the protein band at 70kDa specifically precipitated by mAb $1 \mathrm{H1}$. (C) Western blot analysis of membrane proteins of CEK cells after immunoprecipitation assay. Sample orders were same as shown in (B). (D) Amino acid sequence of HSPA8. The gel fragment indicated with the arrowhead in (B) was analyzed by LC-MS/MS. The resulting peptide sequences of HSPA8 were underlined and marked as red color. Full length HSPA8 sequence was searched against chicken protein database in Uniprot. (E) Western blot analysis of HSPA8 present in the membrane protein extracted from CEK cells. Membrane protein fraction of CEK cells and whole cell lysates of CEK cells were immunoblotted with antibodies against the cytoplasmic protein marker (GAPDH), nuclear protein marker (Histone3), membrane protein marker (HSP90AB1) and HSPA8. WCL, whole cell lysates. 
A

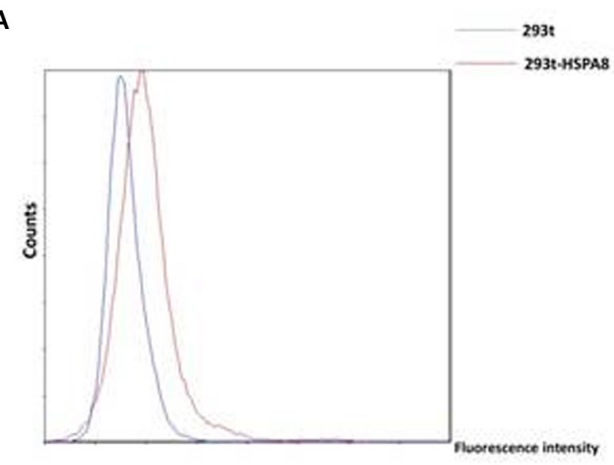

C

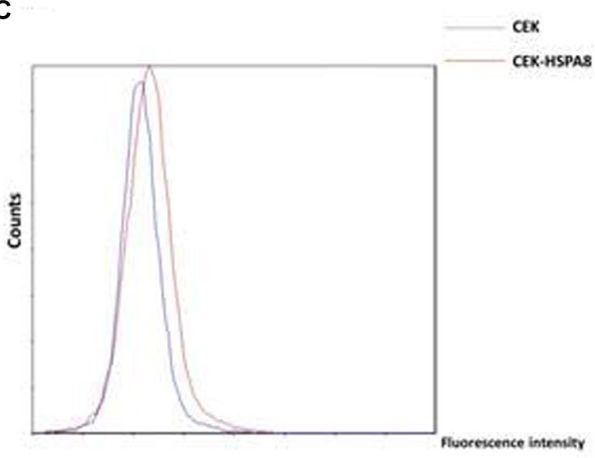

E

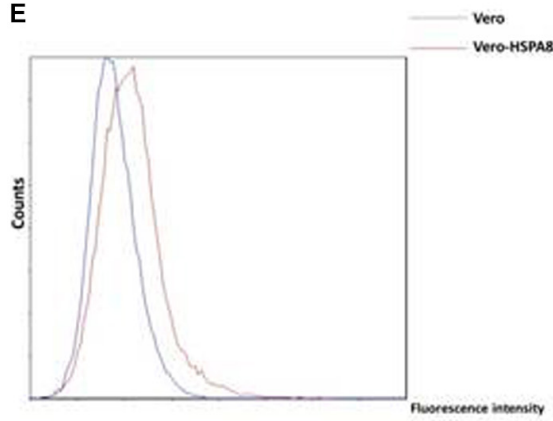

B

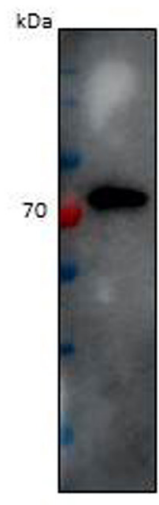

D $k D$

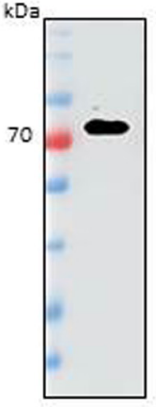

$\mathbf{F}$

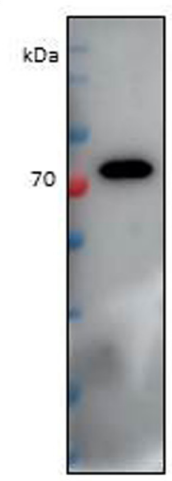

G

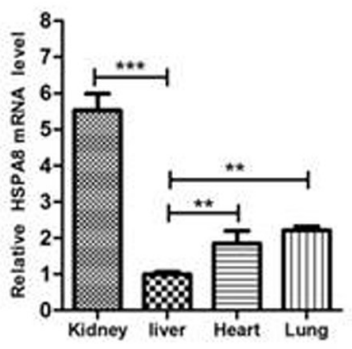

FIGURE 2 | Detection of expression of HSPA8 on cell surface and chicken tissues. (A,C,E) Identification of HSPA8 expression on surface of 293 T cells (A), CEK cells (C) and Vero cells (E) by flow cytometry analysis with antibody against HSPA8. Total $6.5 \times 10^{4}$ of 293T cells, CEK cells and Vero cells were used for each analysis. The blue lines mean without primary antibody and the red lines indicated the reaction with HSPA8 antibody. (B,D,F) Western blot analysis of the membrane protein extracts of 293T cells (B), CEK cells (D) and Vero cells (F) with antibody against HSPA8. (G) Detection of HSPA8 expression level in 1-day-old chicken tissues by RT-qPCR. Tissue samples collected from three chickens were used for the analysis of HSPA8 expression level. ${ }^{\star \star} P<0.01 ;{ }^{* \star *} P<0.001$. 
A

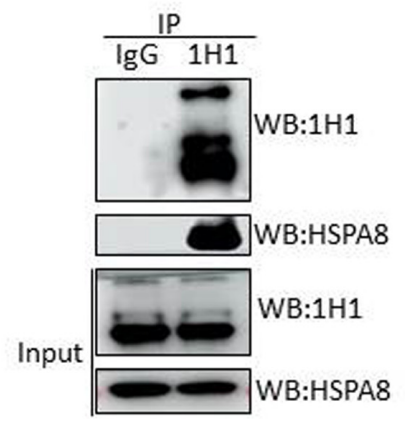

C

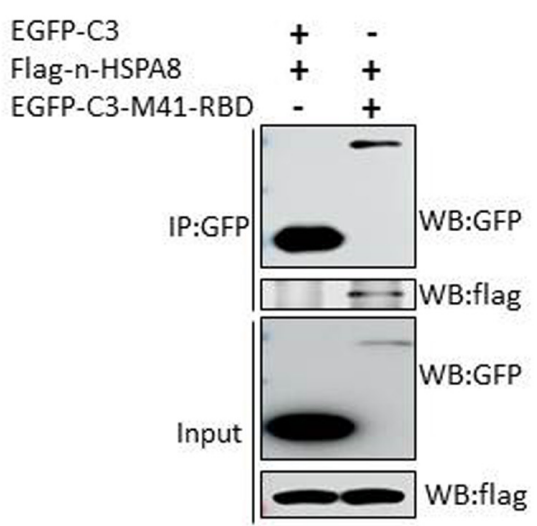

B

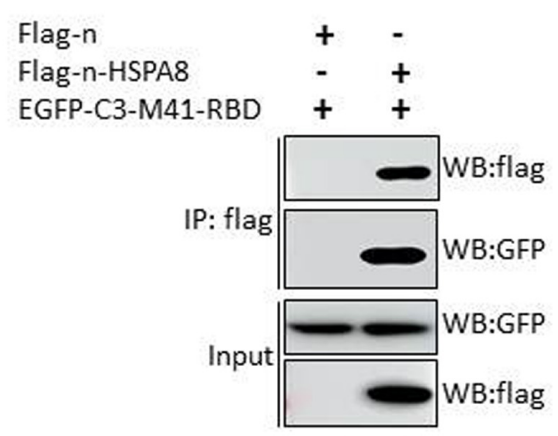

D

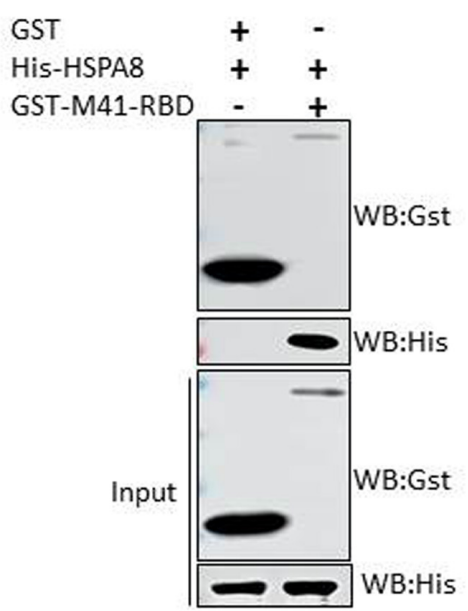

E

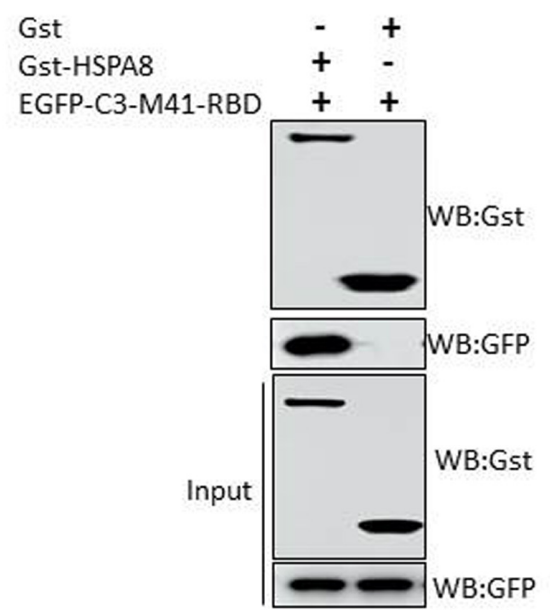

FIGURE 3 | Interaction of HSPA8 with RBD of IBV spike protein. (A) Detection of the interaction between HSPA8 and IBV M41 spike protein in M41-infected CEK cells by immunoprecipitation assay using mAb $1 \mathrm{H} 1$ to IBV $\mathrm{S}$ and mouse IgG. IBV S1 and HSPA8 in precipitates were detected by immunoblots with mAbs to IBV S1 and HSPA8. (B) Detection of the interaction between HSPA8 and IBV M41-RBD by co-IP assay using HSPA8 as bait protein. 293T cells were cotransfected with indicated plasmids and then flag-tagged HSPA8 was immunoprecipitated with anti-flag antibody. The precipitated proteins were detected by Western blotting using anti-flag and anti-GFP antibodies. (C) Detection of the interaction between HSPA8 and IBV M41-RBD by reciprocal co-IP assay using M41-RBD as bait protein. 293T cells were cotransfected with indicated plasmids and then GFP-tagged M41-RBD was immunoprecipitated with anti-GFP antibody. The precipitated proteins were detected by Western blotting using anti-flag and anti-GFP antibodies. (D,E) Identification of the direct interaction between HSPA8 and IBV M41-RBD by GST-pull-down assay. In (D), GST and GST-M41-RBD recombinant proteins were separately bound to glutathione-sepharose beads and then incubated with His-HSPA8 recombinant protein. The protein complexes were detected by Western blot analysis with antibodies against GST and 6xHis. In (E), The lysates of 293T cells transfected with EGFP-C3-M41-RBD vector were incubated with GST or GST-HSPA8 protein which was separately bound to glutathione-sepharose beads. Subsequently, the protein complexes were detected by Western blot analysis with antibodies against GST and GFP. 


\section{HSPA8 Interacts With RBD of IBV Spike Protein}

To further verify the interaction between IBV M41 spike protein and HSPA 8 of host cell, we performed immunoprecipitation assay with $\mathrm{mAb} 1 \mathrm{H} 1$ to spike protein in M41 infected CEK cells. As shown in Figure 3A, HSPA8 could be immunoprecipated with IBV spike protein, but not with mouse IgG. These results indicated that HSPA 8 could interact with IBV spike protein. To examine whether HSPA8 interacts with spike protein through the RBD, we performed co-IP assay with lysates of transfected cells. As shown in Figure 3B, M41-RBD specifically immunoprecipitated with HSPA8, but not with the empty vector control. To further confirm the interaction between HSPA8 and M41-RBD, reciprocal co-IP was performed using M41$\mathrm{RBD}$ as bait protein. The results showed that HSPA8 could be immunoprecipitated with M41-RBD, but not with the empty vector control (Figure 3C).

Next, in vitro GST-pull-down assay was also performed to further confirm the direct interaction between HSPA8 and
M41-RBD. The results indicated that HSPA8 could specifically interact with M41-RBD, but not with the control GST protein (Figures 3D,E).

\section{Recombinant HSPA8 Protein and Anti-HSPA8 Antibody Block/Inhibit IBV Infection of CEK Cells}

Binding blocking assay was performed using recombinant HSPA8 as competitor to confirm its role in IBV infection. As shown in Figure 4A, the amount of viral RNA associated with cells following incubation at $4^{\circ} \mathrm{C}$ with virus pre-incubated with HSPA8 was less than the amount of viral RNA associated with cells incubated with virus pre-incubated with GST protein.

In addition, an inhibition assay was also performed to check whether antibody to HSPA8 could inhibit IBV M41 infection of CEK cells. As shown in Figures 4B,C, infection of IBV was significantly reduced in anti HSPA8 polyclonal antibody pretreated cells at both 1 hpi and 24 hpi. A similar result was also observed in Western blot analysis at 24hpi

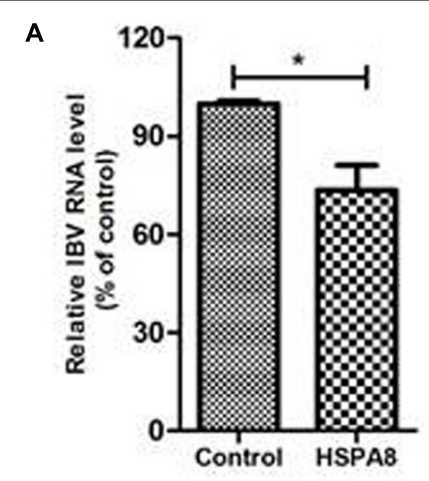

C

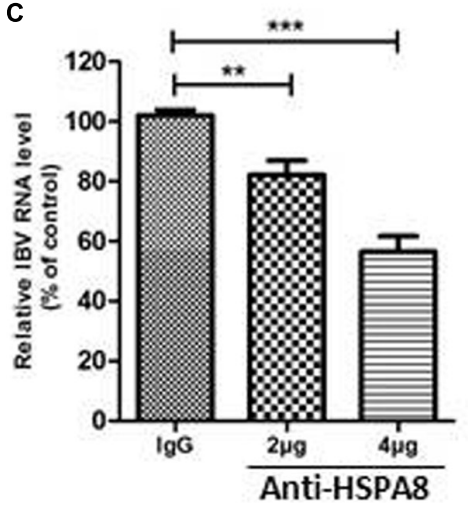

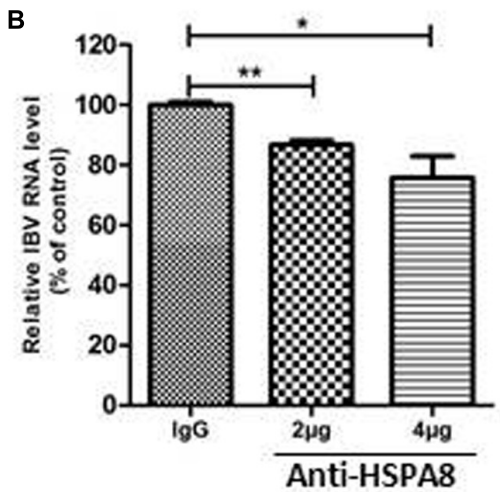

D

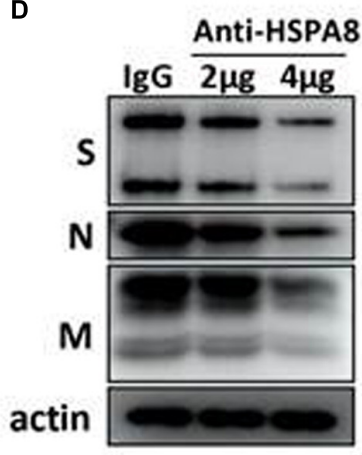

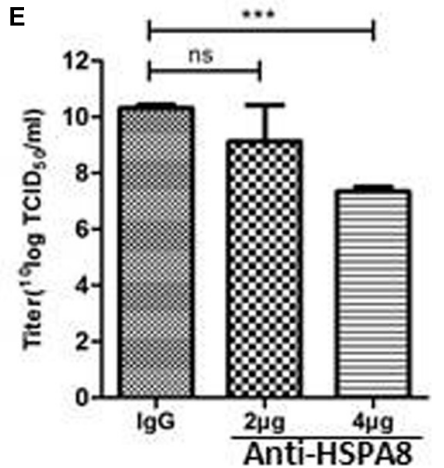

FIGURE 4 | Inhibition of IBV M41 binding to CEK cells by binding blocking assay and infection inhibition assay. (A) Recombinant HSPA8 blocked the binding of IBV M41 to CEK cells. IBV M41 $\left(T_{C I D}=10^{6.5} / \mathrm{ml}, 200 \mu \mathrm{l}\right)$ was incubated with recombinant protein GST-HSPA8 (100 $\left.\mu \mathrm{g}\right)$ and control recombinant protein GST (100 $\mu \mathrm{g})$ at $37^{\circ} \mathrm{C}$ for $1 \mathrm{~h}$. CEK cells cultured in 6-well plates was incubated with GST-HSPA8-treated virus and GST-treated virus at $4^{\circ} \mathrm{C}$ for $1 \mathrm{~h}$. After $1 \mathrm{~h}$ incubation, the cells were harvested after 3 washes with PBS. The viral RNA associated with cells was determined by RT-qPCR. (B-E) Anti HSPA8 antibody inhibited IBV infection in CEK cells. CEK cells were cultured in 6-well plates and incubated with polyclonal antibody against HSPA8 $(2 \mu \mathrm{g}, 2 \mu \mathrm{l} / \mathrm{well}$ or $4 \mu \mathrm{g}, 4 \mu \mathrm{l} / \mathrm{well})$ and rabbit-lgG $(4 \mu \mathrm{g}, 4$ $\mu \mathrm{l} /$ well) at $37^{\circ} \mathrm{C}$ for $1 \mathrm{~h}$. The cells were then incubated with IBV M41 virus $\left(\mathrm{TCID}_{50}=10^{6.5} / \mathrm{ml}, 50 \mu \mathrm{l} /\right.$ well) at $4^{\circ} \mathrm{C}$ for $1 \mathrm{~h}$ then incubated at $37^{\circ} \mathrm{C}$. Cells were harvested after 3 washes with PBS at $1 \mathrm{hpi}$ (B) and 24 hpi (C,D). The inhibition level was determined by RT-qPCR (B,C) and Western-blotting (D). Virus titer in culture supernatant at $24 \mathrm{hpi}$ was determined by $\mathrm{TCID}_{50}$ assay $(\mathbf{E})$. Data shown in this figure represent those from three independent experiments. ${ }^{*} P<0.05$; ${ }^{* *} P<0.01$; ${ }^{* \star *} P<0.001 ;$ ns (nonsignificant), $P>0.05$. 
(Figure 4D). Virus titer in infected cell culture supernatant at $24 \mathrm{hpi}$ from the anti HSPA8 polyclonal antibody pretreated cells was lower than in that from control antibody pretreated cells (Figure 4E). Previous studies have shown that expression of functional cellular receptor in nonpermissive cells could make the cell susceptible to virus infection (Zhang et al., 2018; Wang and Huang, 2019). However, IBV M41 could not be passaged in an HSPA8-overexpressing cell line (Supplementary Figure 1).

\section{HSPA8 Interacts With the N-Terminal 19-272 Amino Acids of S1 of Other IBV Strains and M41-RBD Interacts With HSPA8 of Human and Pig}

Apart from the M41-RBD, we also performed similar experiments with the N-terminal 19-272 amino acids of the S1 subunit of other IBV strains. The results showed that the HSPA8 could interact with the N-terminal 19-272 amino acids of S1 of IBV Beaudette, H120 and QX strains (Figures 5A-C).
Surprisingly, HSPA8 from human and pig also interacted with M41-RBD as indicated by co-IP assay (Figure 5D).

\section{HSPA8 Plays a Role in IBV Beaudette Infection of Vero Cells}

IBV-Beaudette strain could bind to heparan sulfate (HS) through its HS binding site in S2 subunit (Madu et al., 2007). To exclude the influence of HS on the surface of Vero cells for IBV Beaudette binding, heparanase I was used to remove $\mathrm{HS}$ on the surface of Vero cells. As shown in Figure 6A, the viral RNA associated with cells was significantly reduced in heparanase I-pretreated cells compared to the control cells. These results indicated that pretreatment with heparanase I could inhibit IBV Beaudette binding to Vero cells. Further, the importance of HSPA8 in IBVBeaudette strain infection was also studied by binding blocking assay and infection inhibition assay after removal of HS with heparanase I. As shown in Figure 6B, when HS was removed, recombinant HSPA8 was able to block IBV Beaudette strain binding to Vero cells. In the case of infection inhibition assay,
A

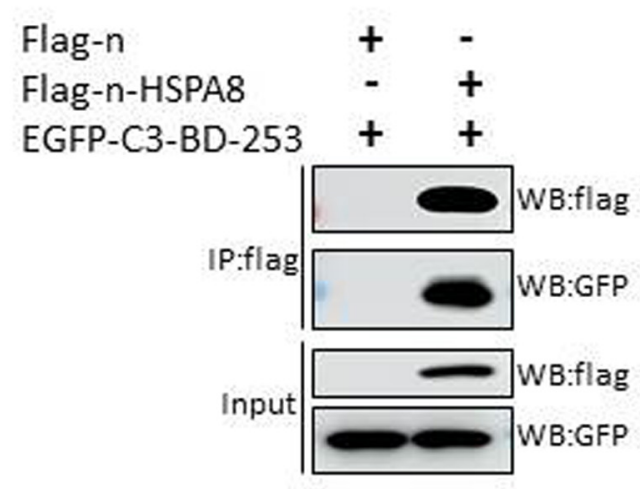

C

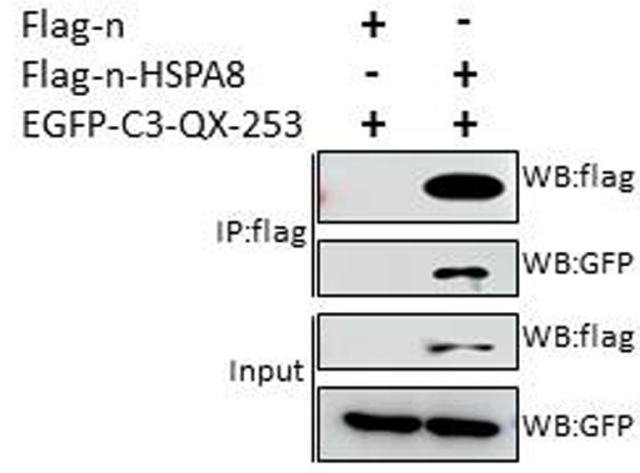

B

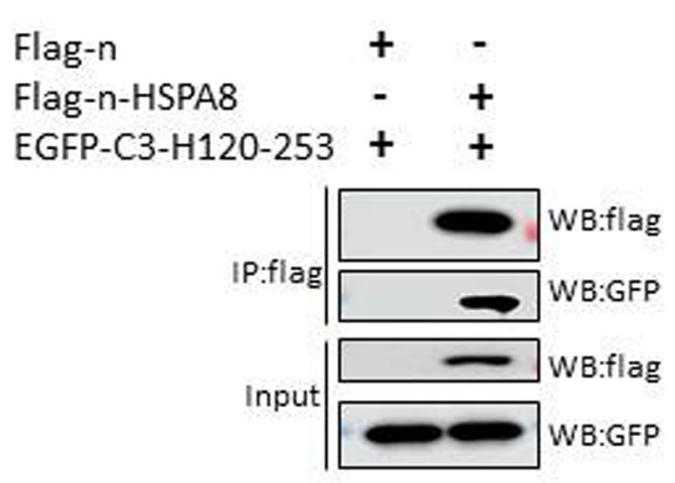

D

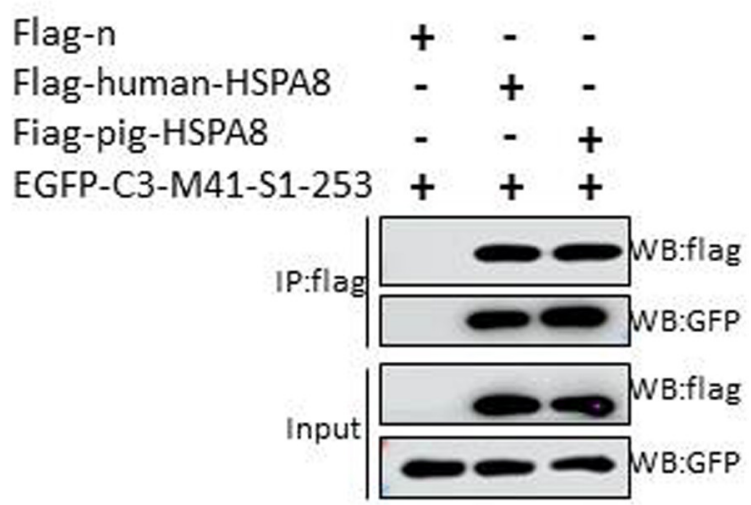

FIGURE 5 | HSPA8 interacts with the N-terminal 19-272 amino acids of S1 subunit of IBV Beaudette, H120 and QX strains and M41-RBD interacts with HSPA8 of human and pig. (A-C) Detection of interaction between chicken HSPA8 and the N-terminal 19-272 amino acids of the S1 subunit of Beaudette (A), H120 (B), and QX (C) strains. 293T cells were cotransfected with indicated plasmids and then flag-tagged HSPA8 was immunoprecipitated with anti-flag antibody. The precipitated proteins were detected by Western blotting using anti-flag and anti-GFP antibodies. (D) Detection of the interaction between IBV M41-RBD and HSPA8 of human and pig. 293T cells were cotransfected with indicated plasmids and then flag-tagged HSPA8 was immunoprecipitated with anti-flag antibody. The precipitated proteins were detected by Western blotting using anti-flag and anti-GFP antibodies. 
the amount of viral RNA was significantly reduced in HSPA8 antibody pretreated cells compared to the control IgG pretreated cells (Figures 6C,D). Similar result was observed in Western blot analysis which showed that infection of IBV was significantly inhibited by HSPA8 antibody compared to the control IgG (Figure 6E). Virus titer in infected cell culture supernatant from the anti HSPA8 antibody pretreated cells was also lower than control antibody pretreated cells (Figure 6F).

\section{DISCUSSION}

In this study, we performed immunoprecipitation assay with membrane proteins from IBV M41 infected CEK cells and found that HSPA8 was involved in the interaction of host cell membrane and IBV spike protein (Figure 1). Then, we confirmed the expression of HSPA8 on the plasma membrane of different cell types by indirect flow cytometry assay (Figures $\mathbf{2 A}, \mathbf{C}, \mathbf{E}$ ), and by Western blot analysis (Figures 2B,D,F). The result of immunoprecipitation and prevalence of HSPA8 on host cell membrane suggested its putative role during initial stages of virus infection. Interestingly, the expression level of HSPA8 in kidney and lung tissues of chicken is higher compared to other organs (Figure 2G). These results suggest that HSPA8 might be involved in tissue tropism of IBV infection in chicken, considering that IBV infection usually causes respiratory distress and kidney damage.

$\mathrm{RBD}$ of coronavirus $\mathrm{S} 1$ protein is primarily responsible for virus interaction with host cells. The N-terminal 19-272 amino acids of the S1 subunit of IBV-M41 was identified as a RBD (Promkuntod et al., 2014). We found that HSPA8 directly interacted with the RBD of IBV M41-spike protein as confirmed by GST-pull-down assay (Figure 3). Furthermore, HSPA8 also interacted with the RBD-associated N-terminal 19-272 amino acids region of the S1 subunit of IBV Beaudette, H120 and QX strains (Figure 5). Binding blocking assay and infection
A

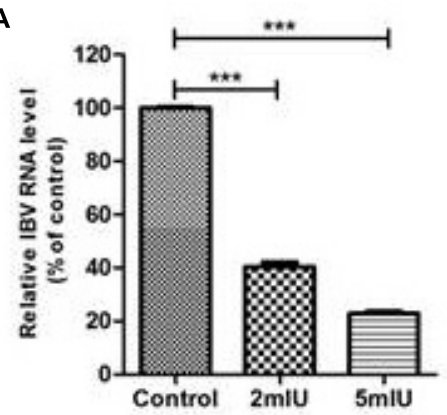

D

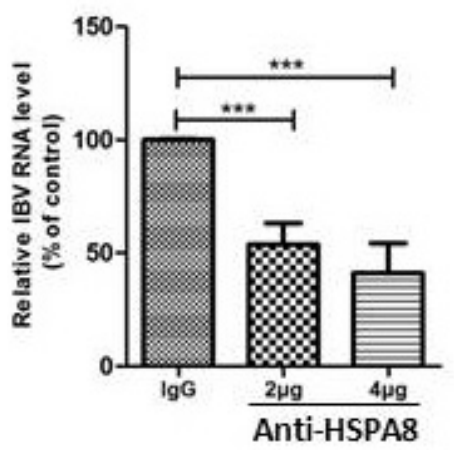

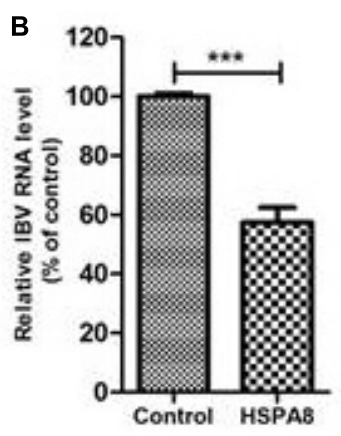

E

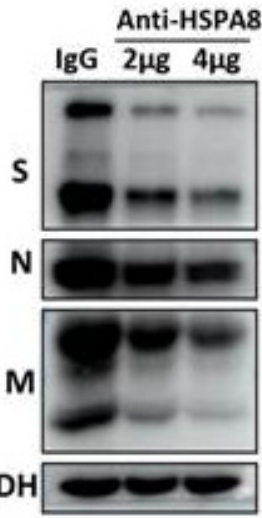

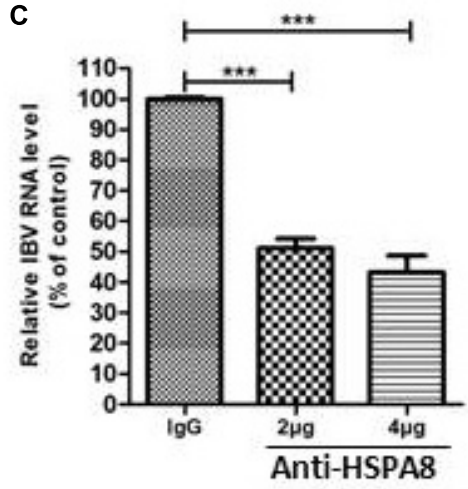

F

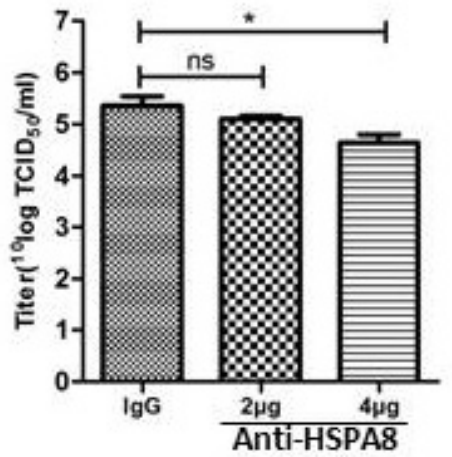

FIGURE 6 | Inhibition of IBV Beaudette infection in Vero cells by binding blocking assay and infection inhibition assay. (A) Confirmation of the effects of removal of HS on IBV Beaudette infection. Before infection, $400 \mu \mathrm{l}$ of heparinase I at different concentration $(0,2,5 \mathrm{mlU} / \mathrm{ml}) \mathrm{was}$ added to Vero cells at $37^{\circ} \mathrm{C}$ for $1 \mathrm{~h}$. The cells were then incubated with IBV Beaudette strain $(\mathrm{moi}=1)$ at $4^{\circ} \mathrm{C}$ for $1 \mathrm{~h}$. After incubation, the cells were harvested after 3 washes with PBS. The viral RNA associated with cells was determined by RT-qPCR. (B) Recombinant HSPA8 blocked the binding of IBV Beaudette to Vero cells. IBV Beaudette (moi = 10) was incubated with recombinant protein GST-HSPA8 $(100 \mu \mathrm{g})$ or control protein GST $(100 \mu \mathrm{g})$ at $37^{\circ} \mathrm{C}$ for $1 \mathrm{~h}$ and then added to Vero cells pretreated with $400 \mu \mathrm{l}$ of heparanase I $(5$ $\mathrm{mlU} / \mathrm{ml}$ ) and incubated at $4^{\circ} \mathrm{C}$ for $1 \mathrm{~h}$. The cells were harvested after 3 washes with PBS. The viral RNA associated with cells was quantified by RT-qPCR. (C-F) Anti-HSPA8 antibody inhibited IBV-Beaudette infection of Vero cells. Vero cells pretreated with $400 \mu \mathrm{l}$ of heparanase I (5 mIU/ml) were incubated with polyclonal antibody against HSPA8 ( $2 \mu \mathrm{g}, 2 \mu \mathrm{l} /$ well or $4 \mu \mathrm{g}, 4 \mu \mathrm{l} /$ well) and rabbit-lgG ( $4 \mu \mathrm{g}, 4 \mu \mathrm{l} /$ well) at $37^{\circ} \mathrm{C}$ for $1 \mathrm{~h}$. The cells were then incubated with IBV Beaudette strain $(\mathrm{moi}=1)$ at $4^{\circ} \mathrm{C}$ for $1 \mathrm{~h}$ then incubated at $37^{\circ} \mathrm{C}$. Cells were harvested after 3 washes with PBS at $1 \mathrm{hpi}(\mathbf{C})$ and $24 \mathrm{hpi}(\mathbf{C}, \mathbf{D})$. The inhibition level was quantified by RT-qPCR (C,D) and Western-blotting (E). Virus titer in culture supernatant at 24 hpi was determined by TCID50 assay (F). Data shown in this figure represent those from three independent experiments. ${ }^{\star} P<0.05$; ${ }^{\star \star} P<0.01$; ${ }^{\star \star \star} P<0.001$; ns (nonsignificant), $P>0.05$. 
inhibition assay showed that recombinant HSPA8 protein and antibody against HSPA8 could inhibit the infection of IBV M41 and Beaudette strains in CEK and Vero cells, respectively (Figures 4, 6). These results indicate that HSPA8 acts as a target molecule on host cell membrane for IBV spike protein and is involved in IBV infection.

However, HSPA8 overexpression in the nonpermissive cells did not facilitate IBV infection (Supplementary Figure 1) and the HSPA8 antibody did not completely abolish the infection of IBV of the host cells. Considering that many binding molecules on the cell surface are involved in coronavirus infection using different strategies, we hypothesize that the HSPA8 might act as an attachment factor during the IBV infection.

It has been widely known that HSPA8 has multiple functions including as a receptor for virus infection (Sagara et al., 1998; Guerrero and Moreno, 2012). In this study, for the first time, HSPA8 was found to play a role in IBV infection. Zhang et al. (2017) identified HSP70 protein, one of the members of HSP70 family, as a part of the receptor complex for IBV. HSP70, which acts as a receptor associated protein, is different from previously identified carbohydrate like receptors such as $\alpha-2$, 3 sialic acid, and HS, suggesting that IBV could exploit both protein and carbohydrate molecules alike to gain entry into the target cells. HSPA8 and HSP70 both belong to the HSP70 family and their amino acid sequences share $86 \%$ identity. Thus, it is quite possible that HSPA8 exhibits similar function as HSP70 during IBV infection.

HS has been reported to co-localize with heat shock protein (HSP) 90 (Snigireva et al., 2015). To check whether HS on cell membrane may have influenced IBV S protein binding to HSPA8, we performed the binding blocking assay and infection inhibition assay based on removal of HS by using heparanase. The results showed that the recombinant HSPA 8 and antibody to HSPA8 could still block/inhibit IBV infection of host cells, suggesting that HSPA8 plays a role during IBV infection independent from HS (Figures 6B-F).

In this study, we found that unglycosylated RBD which was expressed in E. coli could interact with HSPA8. Recent studies showed that the glycosylation of IBV M41-RBD is essential for its binding to chicken tracheal tissue and the removal of the glycosylation modification site of RBD may affect IBV binding to host cells (Parsons et al., 2019; Bouwman et al., 2020). Considering that HSPA8 is not a functional receptor for IBV and the importance of glycosylation of RBD for binding to host cells, we speculate that unglycosylated $\mathrm{RBD}$ might not act like glycosylated RBD to interact with more host molecules. On the other hand, IBV S1 RBD tends to bind sialylated glycan receptor on the surface of host cells (Bouwman et al., 2020), and it has been reported that HSPA 8 could also be glycosylated

\section{REFERENCES}

Belouzard, S., Millet, J. K., Licitra, B. N., and Whittaker, G. R. (2012). Mechanisms of coronavirus cell entry mediated by the viral spike protein. Viruses Basel 4, 1011-1033. doi: 10.3390/v4061011

Bijlenga, G., Cook, J. K. A., Gelb, J., and de Wit, J. J. (2004). Development and use of the $\mathrm{H}$ strain of avian infectious bronchitis virus from the Netherlands
(Guinez et al., 2010), therefore the glycosylation of HSPA8 may increase the possibility for IBV binding to the host cells.

Finally, in this study, HSPA8 from human and pig was also found to interact with M41-RBD (Figure 5D), and sequence analysis showed that the similarity of amino acid sequence among HSPA8 of human, pig and chicken was more than $96 \%$, indicating that the role of HSPA8 on coronavirus binding might be conserved among different host species.

Taken together, our results suggest that HSPA8 plays an important role during initial stage of IBV infection. The identification of HSPA8 as an attachment factor for IBV infection will increase our understanding of the mechanism of IBV pathogenesis.

\section{DATA AVAILABILITY STATEMENT}

All datasets generated for this study are included in the article/Supplementary Material.

\section{ETHICS STATEMENT}

The animal study was reviewed and approved by the Committee on the Ethics of Animal of Zhejiang University (ZJU20170388).

\section{AUTHOR CONTRIBUTIONS}

ML, JZ, and PZ designed the experiments. PZ, CL, and CF performed the experiments. PZ, ML, and JZ analyzed the data. $\mathrm{HS}$, PX, and YY prepared the reagents. PZ, ML, and NK drafted the manuscript. All authors read and approved the final manuscript.

\section{FUNDING}

This work was supported by the National Key Research and Development Program of China (Grant No. 2016YFD0500800) and the China Agriculture Research System (Grant No. CARS-40-K13).

\section{SUPPLEMENTARY MATERIAL}

The Supplementary Material for this article can be found online at: https://www.frontiersin.org/articles/10.3389/fmicb. 2020.01630/full\#supplementary-material

as a vaccine: a review. Avian Pathol. 33, 550-557. doi: 10.1080/0307945040001 3154

Bouwman, K. M., Habraeken, N., Laconi, A., Berends, A. J., Groenewoud, L., Alders, M., et al. (2020). N-glycosylation of infectious bronchitis virus M41 spike determines receptor specificity. J. Gen. Virol. doi: 10.1099/jgv.0.001408 [Epub ahead of print]. 
Breslin, J. J., Mork, I., Smith, M. K., Vogel, L. K., Hemmila, E. M., Bonavia, A., et al. (2003). Human coronavirus 229E: receptor binding domain and neutralization by soluble receptor at 37 degrees C. J. Virol. 77, 4435-4438. doi: 10.1128/Jvi.77. 7.4435-4438.2003

Casais, R., Dove, B., Cavanagh, D., and Britton, P. (2003). Recombinant avian infectious bronchitis virus expressing a heterologous spike gene demonstrates that the spike protein is a determinant of cell tropism. J. Virol. 77, 9084-9089. doi: 10.1128/Jvi.77.16.9084-9089.2003

Cavanagh, D. (2007). Coronavirus avian infectious bronchitis virus. Vet. Res. 38, 281-297. doi: 10.1051/vetres:2006055

Cavanagh, D., Davis, P. J., Cook, J. K. A., Li, D., Kant, A., and Koch, G. (1992). Location of the amino-acid differences in the S1 spike glycoprotein subunit of closely related serotypes of infectious-bronchitis virus. Avian Pathol. 21, 33-43. doi: 10.1080/03079459208418816

Chu, H., Chan, C. M., Zhang, X., Wang, Y. X., Yuan, S. F., Zhou, J., et al. (2018). Middle East respiratory syndrome coronavirus and bat coronavirus HKU9 both can utilize GRP78 for attachment onto host cells. J. Biol. Chem. 293, 11709-11726. doi: 10.1074/jbc.RA118.001897

Delmas, B., Gelfi, J., Lharidon, R., Vogel, L. K., Sjostrom, H., Noren, O., et al. (1992). Aminopeptidase- $\mathrm{N}$ is a major receptor for the enteropathogenic coronavirus Tgev. Nature 357, 417-420. doi: 10.1038/357417a0

Guerrero, C. A., and Moreno, L. P. (2012). Rotavirus receptor proteins Hsc70 and integrin alphavbeta3 are located in the lipid microdomains of animal intestinal cells. Acta Virol. 56, 63-70. doi: 10.4149/av_2012_01_63

Guinez, C., Mir, A. M., Martin, N., Leprince, D., Michalski, J. C., Vergoten, G., et al. (2010). Arginine 469 is a pivotal residue for the Hsc70-GlcNAc-binding property. Biochem. Biophys. Res. Commun. 400, 537-542. doi: 10.1016/j.bbrc. 2010.08.089

Hu, J. Q., Li, Y. F., Guo, J. Q., Shen, H. G., Zhang, D. Y., and Zhou, J. Y. (2007). Production and characterization of monoclonal antibodies. Zoonoses Public Health 54, 69-77.

Kint, J., Langereis, M. A., Maier, H. J., Britton, P., van Kuppeveld, F. J., Koumans, J., et al. (2016). Infectious bronchitis coronavirus limits interferon production by inducing a host shutoff that requires accessory protein 5b. J. Virol. 90, 7519-7528. doi: 10.1128/Jvi.00627-16

Lai, M. M. C., and Cavanagh, D. (1997). The molecular biology of coronaviruses. Adv. Virus Res. 48, 1-100. doi: 10.1016/S0065-3527(08)60286-9

Li, W. H., Moore, M. J., Vasilieva, N., Sui, J. H., Wong, S. K., Berne, M. A., et al. (2003). Angiotensin-converting enzyme 2 is a functional receptor for the SARS coronavirus. Nature 426, 450-454. doi: 10.1038/nature02145

Liu, T., Daniels, C. K., and Cao, S. S. (2012). Comprehensive review on the HSC70 functions, interactions with related molecules and involvement in clinical diseases and therapeutic potential. Pharmacol. Ther. 136, 354-374. doi: 10.1016/ j.pharmthera.2012.08.014

Luo, Z., and Weiss, S. R. (1998). Roles in cell-to-cell fusion of two conserved hydrophobic regions in the murine coronavirus spike protein. Virology 244, 483-494. doi: 10.1006/viro.1998.9121

Madu, I. G., Chu, V. C., Lee, H., Regan, A. D., Bauman, B. E., and Whittaker, G. R. (2007). Heparan sulfate is a selective attachment factor for the avian coronavirus infectious bronchitis virus beaudette. Avian Dis. 51, 45-51. doi: 10.1637/0005-2086(2007)051[0045:hsiasa]2.0.co;2

Parsons, L. M., Bouwman, K. M., Azurmendi, H., de Vries, R. P., Cipollo, J. F., and Verheije, M. H. (2019). Glycosylation of the viral attachment protein of avian coronavirus is essential for host cell and receptor binding. J. Biol. Chem. 294, 7797-7809. doi: 10.1074/jbc.RA119.007532

Promkuntod, N., van Eijndhoven, R. E., de Vrieze, G., Grone, A., and Verheije, M. H. (2014). Mapping of the receptor-binding domain and amino acids critical for attachment in the spike protein of avian coronavirus infectious bronchitis virus. Virology 448, 26-32. doi: 10.1016/j.virol.2013.09.018

Raj, V. S., Mou, H., Smits, S. L., Dekkers, D. H., Muller, M. A., Dijkman, R., et al. (2013). Dipeptidyl peptidase 4 is a functional receptor for the emerging human coronavirus-EMC. Nature 495, 251-254. doi: 10.1038/nature12005

Sagara, Y., Ishida, C., Inoue, Y., Shiraki, H., and Maeda, Y. (1998). 71-kilodalton heat shock cognate protein acts as a cellular receptor for syncytium formation induced by human T-cell lymphotropic virus type 1. J. Virol. 72, 535-541. doi: 10.1128/jvi.72.1.535-541.1998

Smith, A. E., and Helenius, A. (2004). How viruses enter animal cells. Science 304, 237-242. doi: 10.1126/science.1094823

Snigireva, A. V., Vrublevskaya, V. V., Afanasyev, V. N., and Morenkov, O. S. (2015). Cell surface heparan sulfate proteoglycans are involved in the binding of Hsp90alpha and Hsp90beta to the cell plasma membrane. Cell Adh. Migr. 9, 460-468. doi: 10.1080/19336918.2015.1103421

Stricher, F., Macri, C., Ruff, M., and Muller, S. (2013). HSPA8/HSC70 chaperone protein Structure, function, and chemical targeting. Autophagy 9, 1937-1954. doi: 10.4161 /auto. 26448

Wan, Y., Shang, J., Graham, R., Baric, R. S., and Li, F. (2020). Receptor recognition by novel coronavirus from Wuhan: an analysis based on decade-long structural studies of SARS. J. Virol. 94:e00127-20. doi: 10.1128/JVI.00127-20

Wang, B., and Huang, Y. (2019). Porcine deltacoronavirus engages the transmissible gastroenteritis virus functional receptor porcine aminopeptidase $\mathrm{N}$ for infectious cellular entry. Eur. J. Immunol. 49, 1633-1633.

Wang, H., Yuan, X., Sun, Y. J., Mao, X., Meng, C. C., Tan, L., et al. (2019). Infectious bronchitis virus entry mainly depends on clathrin mediated endocytosis and requires classical endosomal/lysosomal system. Virology 528, 118-136. doi: 10. 1016/j.virol.2018.12.012

Williams, R. K., Jiang, G. S., and Holmes, K. V. (1991). Receptor for Mouse Hepatitis-Virus Is a Member Of the Carcinoembryonic Antigen Family Of Glycoproteins. Proc. Natl. Acad. Sci. U.S.A. 88, 5533-5536. doi: 10.1073/pnas. 88.13.5533

Winter, C., Schwegmann-Wessels, C., Cavanagh, D., Neumann, U., and Herrler, G. (2006). Sialic acid is a receptor determinant for infection of cells by avian Infectious bronchitis virus. J. Gen. Virol. 87, 1209-1216. doi: 10.1099/vir.0. 81651-0

Zhang, S., Hu, W. W., Yuan, L. F., and Yang, Q. (2018). Transferrin receptor 1 is a supplementary receptor that assists transmissible gastroenteritis virus entry into porcine intestinal epithelium. Cell Commun. Signal. 16:69. doi: 10.1186/ s12964-018-0283-5

Zhang, Z., Yang, X., Xu, P., Wu, X., Zhou, L., and Wang, H. (2017). Heat shock protein 70 in lung and kidney of specific-pathogen-free chickens is a receptorassociated protein that interacts with the binding domain of the spike protein of infectious bronchitis virus. Arch. Virol. 162, 1625-1631. doi: 10.1007/s00705017-3280-x

Conflict of Interest: The authors declare that the research was conducted in the absence of any commercial or financial relationships that could be construed as a potential conflict of interest.

Copyright (C) 2020 Zhu, Lv, Fang, Peng, Sheng, Xiao, Kumar Ojha, Yan, Liao and Zhou. This is an open-access article distributed under the terms of the Creative Commons Attribution License (CC BY). The use, distribution or reproduction in other forums is permitted, provided the original author(s) and the copyright owner(s) are credited and that the original publication in this journal is cited, in accordance with accepted academic practice. No use, distribution or reproduction is permitted which does not comply with these terms. 